\title{
Editorial: Behavioral Insights for a Sustainable Energy Transition
}

\author{
Tobias Brosch ${ }^{1 *}$, David Sander ${ }^{1}$ and Martin K. Patel ${ }^{2}$ \\ ${ }^{1}$ Department of Psychology, Swiss Center for Affective Sciences, University of Geneva, Geneva, Switzerland, \\ ${ }^{2}$ Energy Group, Institute for Environmental Sciences and Forel Institute, University of Geneva, Geneva, Switzerland
}

Keywords: behavioral insights, sustainability, energy, consumer, decisions, determinants, interventions

The Editorial on the Research Topic

Understanding the human factor of the energy transition: Mechanisms underlying energyrelevant decisions and behaviors

In December 2015, 195 countries adopted the Paris Agreement, which aims at a substantial reduction of greenhouse gas emissions to keep the increase in global average temperature to $2^{\circ} \mathrm{C}$ or less. The successful implementation of this global energy transition does not only depend on the development of new energy technologies, but also requires major changes in the patterns of individual energyrelated choices and behaviors. The behavioral sciences can thus make important contributions to the energy transition by providing insights into the factors and mechanisms that underlie these behaviors.

To this end, three main challenges need to be addressed: (1) to investigate and systematize the factors that influence energy-relevant choices and behaviors; (2) based on these insights, to develop and test interventions that promote more sustainable behavior; and (3) given that individual behavior takes place in a political and economic context, to integrate behavioral insights into the context of these larger systems. These challenges are taken up in the contributions of this research topic, which cover the fields of psychology, affective science, behavioral economics, economics, sociology, consumer behavior, business science, sociology, and political science. Here, we give an overview of the contributions and discuss some implications and recommendations for a successful energy transition.

Edited and Reviewed by: Simone Bastianoni,

University of Siena, Italy

*Correspondence: Tobias Brosch tobias.brosch@unige.ch

Specialty section: This article was submitted to Energy Systems and Policy, a section of the journal Frontiers in Energy Research

Received: 22 February 2016 Accepted: 22 March 2016

Published: 18 April 2016

Citation:

Brosch T, Sander D and Patel MK (2016) Editorial: Behavioral Insights for a Sustainable Energy Transition.

Front. Energy Res. 4:15. doi: 10.3389/fenrg.2016.00015

\section{UNDERSTANDING THE DETERMINANTS OF SUSTAINABLE BEHAVIOR}

To successfully promote more sustainable choices and behaviors, we first need to understand their determining factors. Steg et al. propose a framework of the different behaviors that are relevant for sustainability at the household level as well as their determinants and potential intervention strategies. They emphasize the need to develop integrative approaches that explain a wide range of energyrelevant behaviors. Brosch et al. discuss the role of affective factors as influences on energy-relevant decisions. They argue that emotions provide useful information that can lead to better decisions and demonstrate that affective factors can explain energy-relevant behavior above and beyond traditional rational-choice models and value-based approaches. Schubert and Stadelmann analyze the energy efficiency gap from a behavioral economics perspective. They illustrate that consumers may not buy energy-efficient durables - despite the economic advantages these may have in terms of lifetime costs - due to information processing limitations. Skatova et al. demonstrate the important influence of other people's behavior on our choices using an experimental collective-risk game that simulates energy consumption of an interdependent group of participants from the UK. To identify promising targets for sufficiency interventions, Moser et al. analyze the social preferences for energy sufficiency 
behaviors across the domains of mobility, heating, and food in a Swiss sample. They show that while participants would, for example, be willing to reduce their living space to conserve energy, they would be very reluctant to give up meat consumption.

The contributions in this section illustrate the importance of going beyond the boundaries of individual disciplines to integrate the multiple factors that influence energy-relevant choices and behaviors. Aspects related to information processing, such as available information, processing biases, and heuristics, affectivemotivational factors, such as attitudes, values, and emotions, and social influences, such as social norms and status considerations, all are critical determinants of sustainable behavior. Moreover, these factors interact with the choice context, e.g., specific aspects of the behavior/technology in question, available options, potential incentives, and barriers. Thus, any understanding of energy-relevant behavior needs to be based on a consideration of the interaction of personal and situational factors.

\section{DEVELOPING AND TESTING INTERVENTION STRATEGIES TO PROMOTE SUSTAINABLE BEHAVIOR}

A thorough understanding of the factors influencing sustainable behavior allows the development of effective intervention strategies. Shalev discusses how insights from social and motivational psychology can be leveraged to motivate individuals to take action against climate change. She emphasizes the importance of integrating sustainable behavior into a goal system that also includes other concerns regarding domains such as health, economy, and education. Sintov and Schultz demonstrate how behavioral insights can be helpful in the context of smart grid systems, which are used by energy utilities to interact with their consumers in real time. Using examples such as demand response programs, time-of-use pricing, and energy feedback, they illustrate how energy utilities can increase the engagement of their end users. Graffeo et al. test a nudge-like intervention based on social norms on a sample of university students from Jerusalem. Varying the degree of information that is revealed about the source of a social norm, they show that the most efficient norms come from sources that are identified as "living in the same neighborhood as the participant," but that the provision of further identifying information (such as the name and a photo of the source) leads to a decrease of the social influence. Klonek and Kauffeld illustrate the potential of motivational interviewing, a persuasion technique that aims at reducing resistance to behavior change in the sustainability domain. Moreover, they provide training materials that can be used for interventions in organizational settings in the supplementary materials. Pahl et al. discuss their experiences from the eViz project, an intervention project that explores the potential of energy visualizations - thermal images visualizing heat escaping from buildings - to motivate people to invest in energy efficiency measures. They show that energy visualizations can attract attention, elicit affective reactions, and furthermore lead to reductions in energy use as well as increased investments in isolation measures.
The contributions in this section illustrate the enormous potential of applying behavioral insights to the context of sustainability interventions. They help understand why some previously used intervention instruments (e.g., purely financial incentives and information provision) may not function optimally due to bounded rationality. More importantly, they point out ways in which better intervention strategies can be developed by taking into account limited information processing (e.g., better energy label design) and the enormous impact of affective motivational and social factors (e.g., interventions based on affective responses or social influence).

\section{BEHAVIORAL INSIGHTS IN THE CONTEXT OF MARKETS AND POLITICAL SYSTEMS}

Energy-relevant choices and behaviors do not take place in isolation, but in the context of markets and political systems, which interact with the more proximal determinants of decision making as discussed above. It is thus important to consider behavioral insights in the context of these larger systems. Krysiak and Weigt review economic models of energy markets with respect to their representation of consumer behavior. They identify gaps in existing approaches and outline possibilities to integrate more detailed and realistic representations of human behavior into these models. Burger et al. merge theoretical perspectives from multiple disciplines into a framework of energy-related consumption behavior with the "situated individual," an individual living in a complex social environment, at its center. The framework comprises multiple levels of behavioral influences as well as potential interventions at the economic and political level and promises to be a useful tool for guiding future interdisciplinary research.

\section{OUTLOOK}

Developing a more sustainable energy use is one of the most pressing tasks facing our planet and its inhabitants. The research topic illustrates the important contributions the behavioral and social sciences can make by providing insights into the mechanisms that underlie our decisions and behaviors. The increasing integration of theoretical and disciplinary perspectives that is illustrated in the contributions assembled here is a welcome development that will result in a more complete toolbox of intervention strategies, contributing to a sustainable energy transition.

\section{AUTHOR CONTRIBUTIONS}

All authors listed have made substantial, direct, and intellectual contribution to the work and approved it for publication.

\section{FUNDING}

This research topic was supported by the activities of SCCER CREST (Swiss Competence Center for Energy Research), with financial support by the Swiss Commission for Technology and Innovation (CTI) under Grant No. 466 KTI.2014.0114. 
Conflict of Interest Statement: The authors declare that the research was conducted in the absence of any commercial or financial relationships that could be construed as a potential conflict of interest.

Copyright (c) 2016 Brosch, Sander and Patel. This is an open-access article distributed under the terms of the Creative Commons Attribution License
(CC BY). The use, distribution or reproduction in other forums is permitted, provided the original author(s) or licensor are credited and that the original publication in this journal is cited, in accordance with accepted academic practice. No use, distribution or reproduction is permitted which does not comply with these terms. 